\title{
MIMO Antenna Array Design with Polynomial Factorization
}

\author{
Wen-Qin Wang, Huaizong Shao, and Jingye Cai \\ School of Communication and Information Engineering, University of Electronic Science and Technology of China (UESTC), \\ Chengdu 611731, China \\ Correspondence should be addressed to Wen-Qin Wang; wqwang@uestc.edu.cn
}

Received 11 March 2013; Revised 6 June 2013; Accepted 7 June 2013

Academic Editor: Yuan Yao

Copyright (C) 2013 Wen-Qin Wang et al. This is an open access article distributed under the Creative Commons Attribution License, which permits unrestricted use, distribution, and reproduction in any medium, provided the original work is properly cited.

One of the main advantages of multiple-input multiple-output (MIMO) antenna is that the degrees-of-freedom can be significantly increased by the concept of virtual antenna array, and thus the MIMO antenna array should be carefully designed to fully utilize the virtual antenna array. In this paper, we design the MIMO antenna array with the polynomial factorization method. For a desired virtual antenna array, the polynomial factorization method can optimally design the specified MIMO transmitter and receiver. The array performance is examined by analyzing the degrees-of-freedom and statistical output signal-to-interference-plus-noise ratio (SINR) performance. Design examples and simulation results are provided.

\section{Introduction}

Multiple-input multiple-output (MIMO) antenna array has received much attention in communication, radar, and navigation systems [1-4]. MIMO idea is not new; its origin in control systems can be traced back to 1970s [5]. The early 1990s saw an emergence of MIMO ideas into the field of wireless communication systems [6-8]. More recently, the ideas of MIMO appear in sensor and radar systems [9-12]. Different from conventional phased-array systems [13], in MIMO antenna systems each antenna transmits a unique waveform, orthogonal to the waveforms transmitted by other antennas, and a matched filter bank is used to extract the orthogonal waveform components. When the orthogonal signals are transmitted from different antennas, the return of each orthogonal signal will carry independent information. The phase difference caused by different transmitting antennas along with the phase differences caused by different receiving antennas can form a new virtual antenna array steering vector. With optimally designed antenna array geometry, we can create a very long array steering vector with a small number of antennas [14, 15]. More importantly, this provides good flexibility and reconfigurability in array configuration, thus enabling a flexible and reconfigurable system.

Although MIMO antenna arrays are widely used in various applications, radar-related application is assumed in this paper. Even so, the rich literature of research in this area appears in threefold: (a) the comparison between conventional phased-array and MIMO array, and the development of MIMO architecture [16-18]; (b) MIMO waveform diversity and transmitter design $[19,20]$; and (c) the system performance and the receiver design for enhanced performance $[21,22]$. In fact, one of the main advantages of MIMO antenna array is that the degrees-of-freedom can be significantly increased by the concept of virtual antenna array, and thus the MIMO antenna array should be carefully designed to fully utilize the virtual antenna array. This paper focuses on the MIMO sparse antenna array design to obtain a uniform virtual array for the system.

Many array design methods have been proposed for conventional phased arrays [23-26]. Specially, linear programming-based algorithms were proposed by $[27,28]$ for reduced sidelobe thinned array design. Genetic algorithm was proposed by [29] to optimally design thin large phased arrays. Simulated annealing-based technique was proposed by [30] to synthesize the position and weight coefficients of a linear array that minimizes peak sidelobes. A hybrid approach combining particle swarm optimization with combinational techniques was proposed by [31] to synthesize planar thinned arrays. Recently a general polynomial factorization-based design of sparse periodic linear arrays was proposed by [32] to design sparse periodic arrays. In 
the method, transmit and receive aperture polynomials are selected such that their product results in a polynomial representing the desired combined transmit/receive effective aperture function.

In this paper, we use the polynomial factorization method proposed in [32] to design MIMO antenna array and comparatively analyze the designed array performance. To obtain the desired virtual MIMO antenna array, we use the polynomial factorization-based method to design the transmit and receive antenna arrays. Since there may be multiple solutions for the transmit and receive antenna arrays and different solutions may have different array performance, we use adaptive beamforming algorithm to comparatively analyze the statistical output signal-to-interference-plus-noise ratio (SINR) performance of the designed arrays. Extensive design examples and simulation results are also provided.

The remaining sections are organized as follows: after introducing the polynomial factorization in Section 2, we describe the polynomial factorization-based MIMO antenna array design in Section 3. Next, the output SINR performance of the designed MIMO antenna arrays is analyzed by adaptive beamforming on the receiver in Section 4, and design examples and simulation results are provided in Section 5. Finally, this paper is concluded in Section 6 with a short discussion of future work.

\section{General Polynomial Factorization Method}

The polynomial factorization method is based on factorizing a polynomial $P_{M}(x)$ of degree $M$ in positive powers of $x$ and with unity coefficients [33]

$$
P_{L}(x)=\sum_{l=0}^{L} x^{l} .
$$

Let the positive integer of the number of coefficients $L+1$ be expressible as a product of $K+1$ irreducible positive integers $L_{k}+1,0 \leq k \leq K$ :

$$
L+1=\prod_{k=0}^{K}\left(L_{k}+1\right) .
$$

Considering the product $P_{L_{0}}(x) P_{L_{1}}\left(x^{S_{0}}\right)$, we have

$$
\begin{aligned}
P_{L_{0}}(x) P_{L_{1}}\left(x^{S_{0}}\right) & \left(\sum_{l=0}^{L_{0}} x^{l}\right)\left(\sum_{l=0}^{L_{1}} x^{l S_{0}}\right) \\
= & \sum_{l=0}^{L_{0}} x^{l}+x^{S_{0}}\left(\sum_{l=0}^{L_{0}} x^{l}\right) \\
& +x^{2 S_{0}}\left(\sum_{l=0}^{L_{0}} x^{l}\right)+\cdots+x^{L_{1} S_{0}}\left(\sum_{l=0}^{L_{0}} x^{l}\right) \\
= & \sum_{l=0}^{L_{1} S_{0}+L_{0}} x^{l} .
\end{aligned}
$$

Therefore, $P_{L}(x)$ can be expressed as $[32,33]$

$$
P_{L}(x)=\prod_{k=0}^{K} P_{L_{k}}\left(x^{S_{k}}\right),
$$

with

$$
S_{k}=\prod_{i=0}^{k}\left(L_{i-1}+1\right),
$$

where $L_{-1}=0$.

The factorization of a specified $L+1$ into a product of irreducible integers can be carried out using Euclid's algorithm [34]. When $L+1$ can be expressed as a power of two, that is, $L+1=3^{v}, P_{L}(x)$ can be factorized as

$$
P_{L}(x)=(1+x)\left(1+x^{2}\right) \cdots\left(1+x^{2^{\nu-1}}\right) .
$$

Note that the solutions to the cases when $L+1$ cannot be expressed as a power of two can be found in [32]. The decomposition in (6) has been used in designing a sparse antenna array with a uniform aperture function [35] and a linearly tapered aperture function [36].

As an example, we suppose $L+1=10$; using Euclid's algorithm we then have

$$
L+1=10=2 \times 5=5 \times 2 .
$$

Then, according to the above polynomial factorization method, we can get

$$
\begin{aligned}
& P_{L}(x) \\
& \quad=\left(x^{0}+x^{1}\right)\left[\left(x^{2}\right)^{0}+\left(x^{2}\right)^{1}+\left(x^{2}\right)^{2}+\left(x^{2}\right)^{3}+\left(x^{2}\right)^{4}\right] \\
& \quad=\left(x^{0}+x^{1}\right)\left(x^{0}+x^{2}+x^{4}+x^{6}+x^{8}\right) \\
& \quad=(1+x)\left(1+x^{2}+x^{4}+x^{6}+x^{8}\right)
\end{aligned}
$$

or

$$
\begin{aligned}
P_{L}(x) & =\left(x^{0}+x^{1}+x^{2}+x^{3}+x^{4}\right)\left[\left(x^{5}\right)^{0}+\left(x^{5}\right)^{1}\right] \\
& =\left(x^{0}+x^{1}+x^{2}+x^{3}+x^{4}\right)\left(x^{0}+x^{5}\right) \\
& =\left(1+x+x^{2}+x^{3}+x^{4}\right)\left(1+x^{5}\right) .
\end{aligned}
$$

That is to say, there may be multiple possible factorizations for a given polynomial.

\section{Polynomial Factorization-Based MIMO Array Design}

Consider a MIMO radar system with a transmitter equipped with $M$ colocated antennas and a receiver equipped with $N$ colocated antennas. Suppose that both the transmit and receive arrays are close to each other in space (possibly the same array) so that they see targets at the same directions. The 
signal received by each antenna is the weighted summation of all the transmitted waveform

$$
x_{n}(t)=\sum_{m=1}^{M} \alpha_{n, m} \phi_{m}(t), \quad m=1,2, \ldots, M ; n=1,2, \ldots, N,
$$

where $x_{n}(t)$ is the received signal at the $n$th antenna, $\phi_{m}(t)$ is the transmitted waveform at the $m$ th antenna, and $\alpha_{n, m}$ is the channel coefficient with the $m$ th antenna as input and the $n$th antenna as output. Suppose $\mathbf{x}(t)=\left[x_{1}(t), x_{2}(t), \ldots, x_{N}(t)\right]$ and $\phi(t)=\left[\phi_{1}(t), \phi_{2}(t), \ldots, \phi_{N}(t)\right]$, and the above equation can be rewritten in a matrix

$$
\mathbf{x}(t)=\sigma_{s}\left[\mathbf{a}^{T}(\theta) \phi(t)\right] \mathbf{b}(\theta)
$$

where $\theta$ is the target direction, $\sigma_{s}$ is the complex-valued reflection coefficient of the focal point $\theta_{s},(\cdot)^{T}$ is the transpose operator, and $\mathbf{a}(\theta)$ and $\mathbf{b}(\theta)$ are the actual transmit and receive steering vectors associated with the direction $\theta$.

When the transmitted waveforms are orthogonal,

$$
\int \phi_{m}(t) \phi_{n}^{*}(t) \mathrm{d} t= \begin{cases}\delta(t), & m=m^{*} \\ 0, & m \neq m^{*}\end{cases}
$$

where ()$^{*}$ denotes a conjugate operator. The radar return due to the $m$ th transmitted waveform can be separated by matched-filtering the received signal with $\phi_{m}(t), m=$ $1,2, \ldots, M$; that is,

$$
\mathbf{s}_{m} \triangleq \int_{T_{0}} \mathbf{x}(t) \phi_{m}^{*}(t) \mathrm{d} t, \quad m=1,2, \ldots, M,
$$

where $T_{0}$ is the radar pulse width. Therefore, there are a total of $M \times N$ independent signals, which can be written as a $M N \times$ 1 virtual data vector

$$
\mathbf{y} \triangleq\left[\mathbf{s}_{1}^{T}, \mathbf{s}_{2}^{T}, \ldots, \mathbf{s}_{M}^{T}\right]^{T}=\sigma_{s} \mathbf{a}(\theta) \otimes \mathbf{b}(\theta),
$$

where $\otimes$ is the Kronecker product; that is, the virtual antenna array is

$$
\mathbf{v}(\theta)=\mathbf{a}(\theta) \otimes \mathbf{b}(\theta) .
$$

Suppose that the $m$ th transmitting antenna is located at $x_{T, m}=(\lambda / 2) u_{m}$ and the $n$th receiving antenna is located at $x_{R, n}=(\lambda / 2) v_{n}$, where $\lambda$ is the wavelength. Considering a farfield point target, the transmitter and receiver steering vectors can be represented, respectively, by

$$
\begin{aligned}
& \mathbf{a}(\theta)=\left[e^{j u_{1} \pi \sin \theta}, e^{j u_{2} \pi \sin \theta}, \ldots, e^{j u_{M} \pi \sin \theta}\right]^{T}, \\
& \mathbf{b}(\theta)=\left[e^{j v_{1} \pi \sin \theta}, e^{j v_{2} \pi \sin \theta}, \ldots, e^{j v_{N} \pi \sin \theta}\right]^{T} .
\end{aligned}
$$

Note that the first antenna of $\mathbf{a}(\theta)$ and $\mathbf{b}(\theta)$, respectively, is the reference antenna. According to (15), we can get

$$
\mathbf{v}(\theta)=\left[\begin{array}{cccc}
e^{j\left(v_{1}+u_{1}\right) \pi \sin \theta} & e^{j\left(v_{1}+u_{2}\right) \pi \sin \theta} & \cdots & e^{j\left(v_{1}+u_{M}\right) \pi \sin \theta} \\
e^{j\left(v_{2}+u_{1}\right) \pi \sin \theta} & e^{j\left(v_{2}+u_{2}\right) \pi \sin \theta} & \cdots & e^{j\left(v_{2}+u_{M}\right) \pi \sin \theta} \\
\vdots & \vdots & \cdots & \vdots \\
e^{j\left(v_{N}+u_{1}\right) \pi \sin \theta} & e^{j\left(v_{N}+u_{2}\right) \pi \sin \theta} & \cdots & e^{j\left(v_{N}+u_{M}\right) \pi \sin \theta}
\end{array}\right] .
$$

The array response in the $n$th receiving antenna for the $m$ th transmitted waveform can be expressed as

$$
v_{m, n}(\theta)=e^{j\left(v_{n}+u_{m}\right) \pi \sin \theta} .
$$

It can be noticed that the MIMO antenna array response is the same as the target response received by a receiving array with $M N$ antennas located at

$$
\left\{x_{T, m}+x_{R, n}\right\}, \quad m=1,2, \ldots, M, n=1,2, \ldots, N .
$$

This $M N$-element array is just the virtual MIMO array. An utmost of $M N$-element virtual array can be obtained by using only $M+N$ physical antenna elements. It is as if we have a receiving array of $M N$ elements.

According to (18), the far-field radiation pattern at an angle $\theta$ away from the broadside of the virtual MIMO array with uniform weighting is expressed as

$$
\begin{aligned}
P_{V}(\theta) & =\sum_{m=1}^{M} \sum_{n=1}^{N} e^{j\left(v_{n}+u_{m}\right) \pi \sin \theta} \\
& =\sum_{m=1}^{M} e^{j u_{m} \pi \sin \theta} \sum_{n=1}^{N} e^{j v_{n} \pi \sin \theta} \\
& =P_{T}(\theta) P_{R}(\theta),
\end{aligned}
$$

where $P_{T}(\theta)=\sum_{m=1}^{M} e^{j u_{m} \pi \sin \theta}$ and $P_{R}(\theta)=\sum_{n=1}^{N} e^{j v_{n} \pi \sin \theta}$ denote, respectively, the far-field radiation pattern for the transmitter and receiver. For a more general format, by substituting $x=e^{j 2 \pi \sin \theta d / \lambda}$ with $d$ being the interelement spacing, the virtual array, transmit array, and receive array radiation patterns can be expressed, respectively, as

$$
\begin{aligned}
P_{V}(x) & =\sum_{i=0}^{M+N-1} w_{V}(i) x^{i}, \\
P_{T}(x) & =\sum_{i=0}^{M-1} w_{T}(i) x^{i}, \\
P_{R}(x) & =\sum_{i=0}^{N-1} w_{R}(i) x^{i},
\end{aligned}
$$

where $w_{V}(i), w_{T}(i)$, and $w_{V}(i)$ denote the element weighting functions for the virtual array, transmit array, and receive array, respectively. It follows also that [32]

$$
P_{V}(x)=P_{T}(x) P_{R}(x) .
$$

Therefore, the far-field radiation pattern of the virtual array is equal to the product of the transmitter radiation pattern and receiver radiation pattern. This means that we can use different transmit and receive arrays with a combined virtual MIMO array that is a close equivalent to that of a single full array with no missing elements. To reach this aim, like the general polynomial factorization-based design of sparse periodic linear array discussed in [32], we can design the transmit and receive arrays based on factorizing the desired 


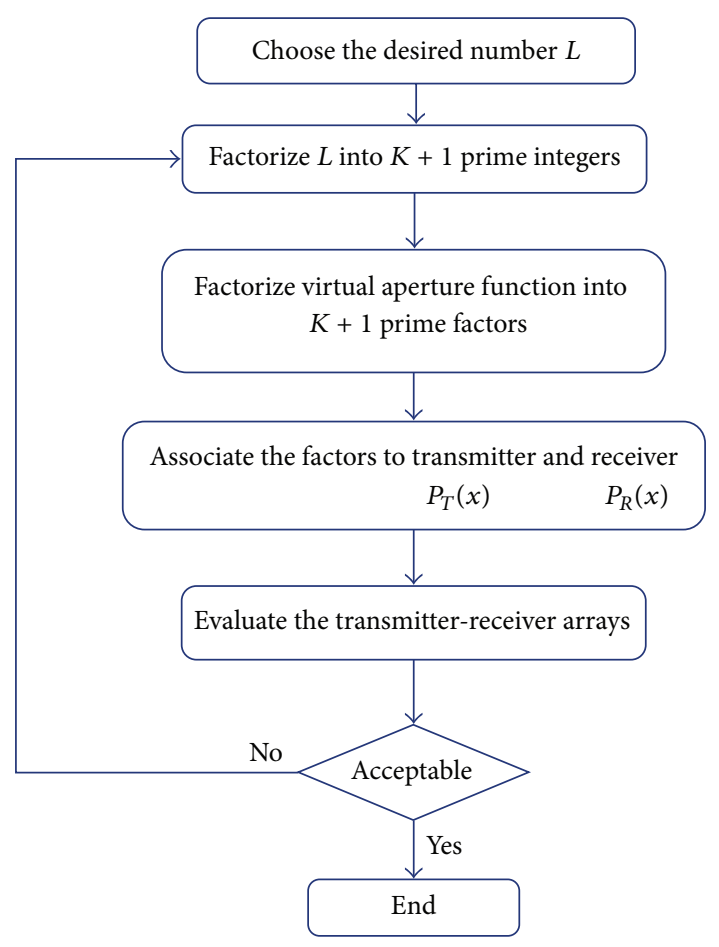

FIgURE 1: Illustration of the MIMO antenna array design steps.

MIMO virtual array polynomial $P_{V}(x)$ as a product of two lower order polynomials with some zero-valued coefficients. In this paper, we aim to optimally design the transmit and receive arrays for a given virtual MIMO antenna array. The design steps are illustrated in Figure 1.

\section{Array Performance Analysis by Adaptive Beamforming}

The output of the matched filter of the MIMO array system can be written as

$$
\mathbf{y}=\alpha_{0} \mathbf{v}(\theta)+\mathbf{n},
$$

where $\mathbf{n}$ denotes the noise plus interference. The output SINR of the beamformer directly impacts the MIMO array performance. The minimum variance distortionless response (MVDR) [37] is a widely employed beamformer choice. The MDVR beamformer for a look angle $\theta$ is given by

$$
\mathbf{w}(\theta)=\frac{\mathbf{R}^{-1} \mathbf{v}(\theta)}{\mathbf{v}^{H}(\theta) \mathbf{R}^{-1} \mathbf{v}(\theta)},
$$

where $(\cdot)^{H}$ is the conjugate transpose and $\mathbf{R}$ is the covariance matrix of the noise and interference signal. The beamformer output can then be expressed as

$$
z=\mathbf{w}^{H}(\theta) \mathbf{y}=\alpha_{0}+\mathbf{w}^{H}(\theta) \mathbf{n} .
$$

Correspondingly, the output SINR can be expressed as

$$
\eta \triangleq \frac{\left|\alpha_{0}\right|^{2}}{\mathbf{w}^{H}(\theta) \mathbf{R} \mathbf{w}(\theta)} .
$$

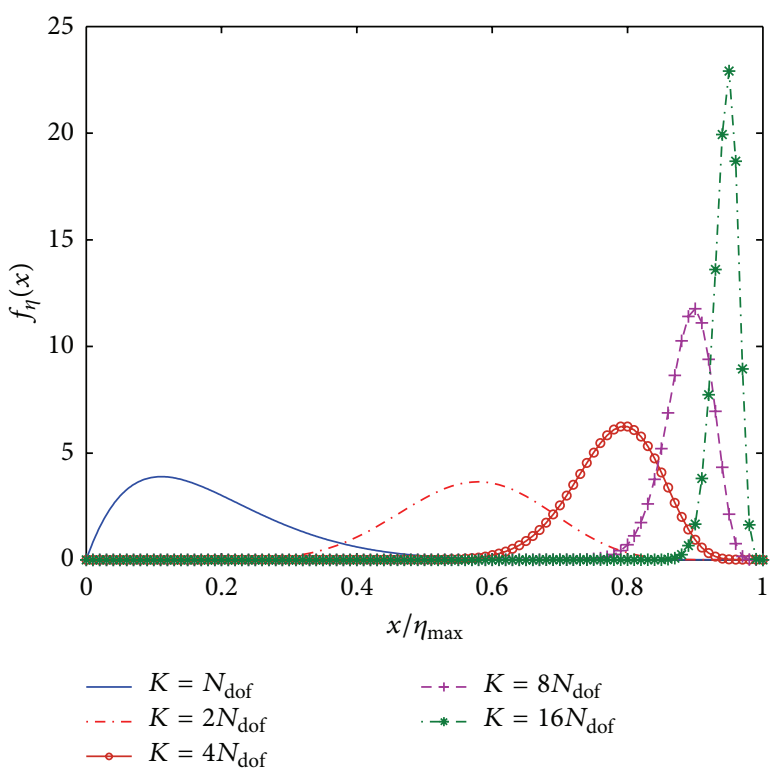

FIgURE 2: The PDF $f_{\eta}(x)$ as a function of $x / \eta_{\max }$.

Since the output SINR is random due to the random covariance matrix $\mathbf{R}$, we can examine the SINR distribution under the standard assumption that $\mathbf{n}$ has a complex Gaussian distribution with zero mean and covariance matrix $\mathbf{R}$. In this case, the probability density function (PDF) of SINR can be represented by $[38,39]$

$$
\begin{aligned}
f_{\eta}(x)= & \frac{K !}{\left(N_{\mathrm{dof}}-2\right) !\left(K+1-N_{\mathrm{dof}}\right) ! \eta_{\max }} \\
& \times\left(1-\frac{x}{\eta_{\max }}\right)^{N_{\mathrm{dof}}-2}\left(\frac{x}{\eta_{\max }}\right)^{K+1-N_{\mathrm{dof}}}, \quad 0 \leq x \leq \eta_{\max },
\end{aligned}
$$

where $K$ is the number of the training signals for obtaining the covariance matrix, $N_{\text {dof }}$ is the number of the system degrees-of-freedom, and $\eta_{\max }$ is the maximum output SINR which is achieved when the covariance matrix is known exactly

$$
\eta_{\max }=\left|\alpha_{0}\right|^{2} \mathbf{v}^{H}(\theta) \mathbf{R}^{-1} \mathbf{v}(\theta) .
$$

Figure 2 shows the $\operatorname{PDF} f_{\eta}(x)$ as a function of $x / \eta_{\max }$.

The detailed derivation of (27) can be found in the appendix of [38]. The corresponding cumulative distribution function is

$$
\begin{aligned}
F_{\eta}(x)= & \frac{K !}{\left(N_{\mathrm{dof}}-2\right) !\left(K+1-N_{\mathrm{dof}}\right) !} \\
& \times \Gamma\left(\frac{x}{\eta_{\max }} ; K+2-N_{\mathrm{dof}}, N_{\mathrm{dof}}-1\right),
\end{aligned}
$$

where $\Gamma(x ; a, b)$ is the incomplete Beta function

$$
\Gamma(x ; a, b) \triangleq \int_{0}^{x} t^{a-1}(1-t)^{b-1} \mathrm{~d} t .
$$




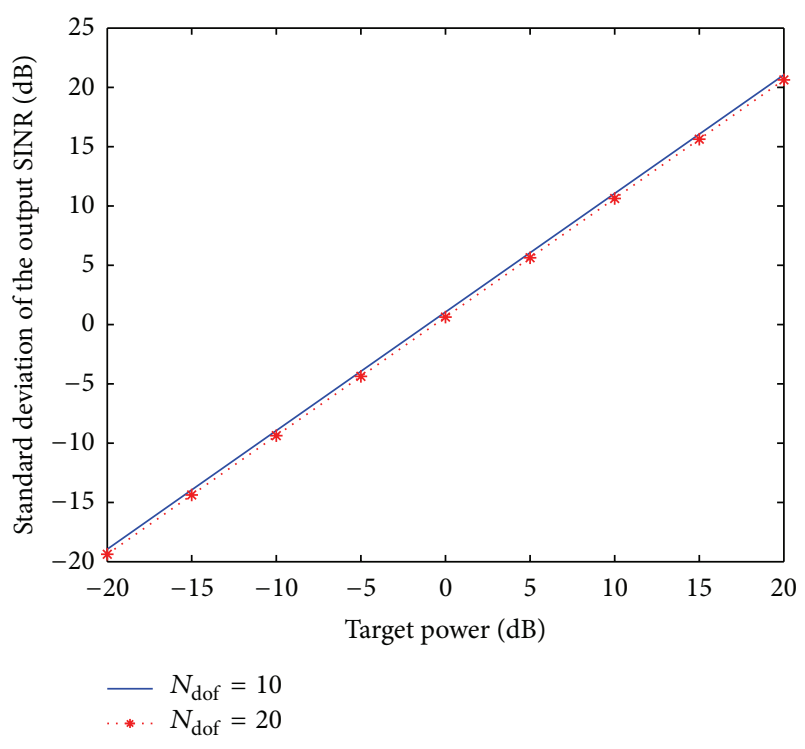

FIGURE 3: Comparative STD of the output SINR performance.

The expectation and variance of the output SINR can be derived, respectively, as

$$
\begin{gathered}
E(\eta)=\eta_{\max } \frac{K+2-N_{\mathrm{dof}}}{K+1}, \\
\operatorname{var}(\eta)=\eta_{\max }^{2} \frac{\left(N_{\mathrm{dof}}-1\right)\left(K+2-N_{\mathrm{dof}}\right)}{(K+2)(K+1)^{2}} .
\end{gathered}
$$

It can be noticed from (31) that the expectation of the output SINR depends mainly on the ratio $N_{\text {dof }} / K$, not the number of the training data, $K$.

Equations (31) and (32) can be used to evaluate the designed MIMO array performance. Figure 3 gives the comparative standard deviation (STD) of the output SINR performance, where $K=20$ is assumed. It can be noticed that, for a given number of the training signals, more degrees-offreedom mean a better STD of the SINR performance.

Define

$$
E(\eta)=\alpha \eta_{\max }
$$

The corresponding $K$ is

$$
K=\frac{1}{1-\alpha}\left(N_{\text {dof }}-2+\alpha\right) \approx \frac{N_{\text {dof }}}{1-\alpha} .
$$

To ensure that the mean output SINR of the adaptive beamformer is within $3 \mathrm{~dB}$ from its maximum achievable value, the training signals should be satisfactory with [38]

$$
K \geq 2 N_{\text {dof }}-3 .
$$

If we want to achieve $\alpha=0.95$, the $K$ should be

$$
K \geq 3 N_{\text {dof }}
$$

\section{Design Examples and Performance Analysis}

We consider two design examples with given virtual MIMO antenna arrays.

Example 1. $L=9$ and $P_{V}(x)=\sum_{i=0}^{9} x^{i}$.

According to (2), we factorize $L+1=10$ into two prime integers 2 and 5 , that is, $10=2 \times 5$. Then, according to (4), the virtual array radiation pattern $P_{V}(x)$ can then be factorized as

$$
P_{V}(x)=(1+x)\left(1+x^{2}+x^{4}+x^{6}+x^{8}\right) .
$$

We can use the first factor for the transmit array and the second factor for the receive array or the first factor for the receive array and the second factor for the transmit array; that is,

$$
\begin{gathered}
P_{T}(x)=1+x, \\
P_{R}(x)=1+x^{2}+x^{4}+x^{6}+x^{8}
\end{gathered}
$$

or

$$
\begin{gathered}
P_{T}(x)=1+x^{2}+x^{4}+x^{6}+x^{8}, \\
P_{R}(x)=1+x .
\end{gathered}
$$

Suppose that the number of the training signals is $K=25$; Figure 4 shows the possible two transmit-receive arrays. It can be noticed that 7 antennas are required for the MIMO antenna arrays and the number of the system degrees-offreedom is 10. Figure 5 gives the statistical mean and STD of the output SINR performance. Note that, although the two arrays have the same statistical SINR performance (because they have the same number of degrees-of-freedom), they should be seen as two different MIMO antenna arrays in the design because they have different transmit array gain and receive array gain (MIMO antenna array has no transmit array gain whereas phased array has both transmit array gain, and receive array gain).

Example 2. $L=11$ and $P_{V}(x)=\sum_{i=0}^{11} x^{i}$.

According to (2), we can factorize $L+1=12$ into the following three ways.

Factorization 1 . We can factorize 12 into three prime integers 2,2 , and 3 , that is, $12=2 \times 2 \times 3$. Then, according to (4), the virtual array radiation pattern $P_{V}(x)$ can then be factorized as

$$
P_{V}(x)=(1+x)\left(1+x^{2}\right)\left(1+x^{4}+x^{8}\right) .
$$

Factorization 2 . We can factorize 12 into three prime integers 2,3 , and 2 , that is, $12=2 \times 3 \times 2$. Then, according to (4), the 


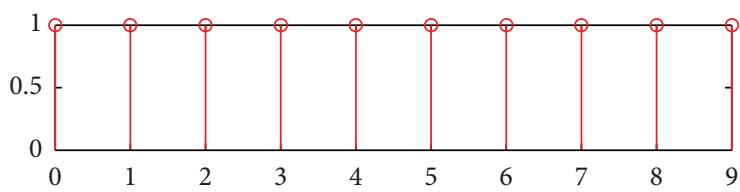

(a) virtual antenna array positions

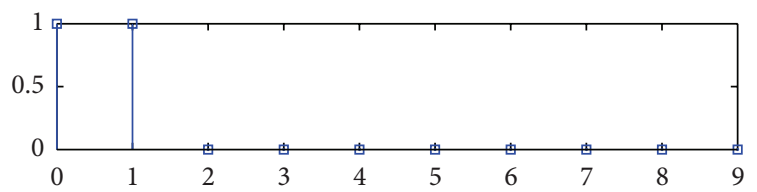

(b) transmit antenna array positions

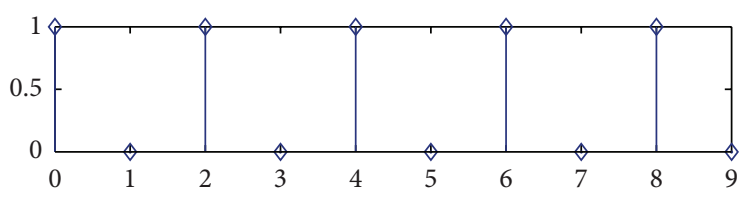

(c) receive antenna array positions

FIGURE 4: Illustration of the designed MIMO antenna array positions. The $x$-axis stands for the MIMO antenna position. $y=1$ denotes an antenna and $y=0$ means no antenna.

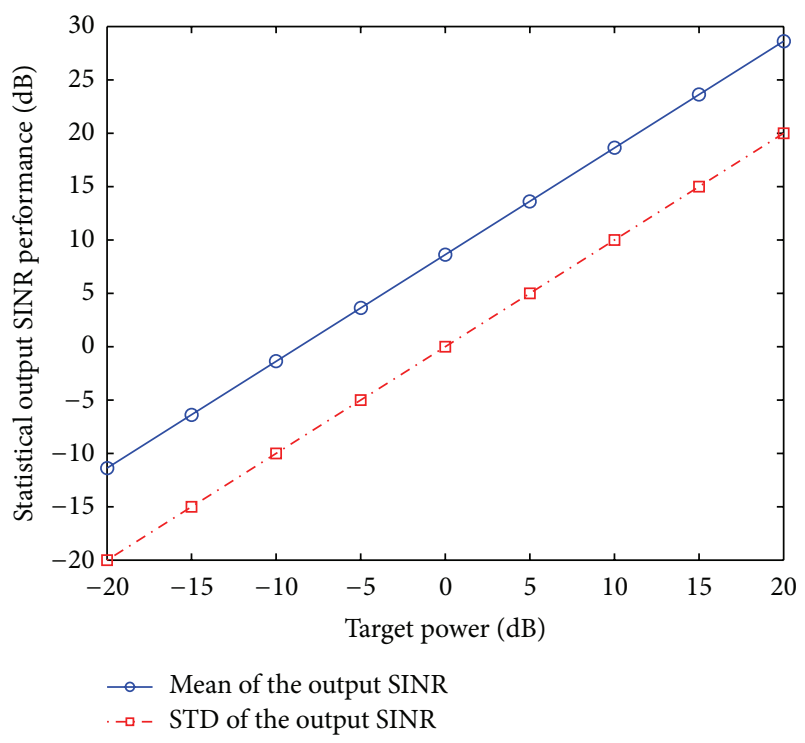

FIGURE 5: Statistical output SINR performance.

virtual array radiation pattern $P_{V}(x)$ can then be factorized as

$$
P_{V}(x)=(1+x)\left(1+x^{2}+x^{4}\right)\left(1+x^{6}\right) .
$$

Factorization 3. We can factorize 12 into three prime integers 3,2 , and 2 , that is, $12=3 \times 2 \times 2$. Then, according to (4), the virtual array radiation pattern $P_{V}(x)$ can then be factorized as

$$
P_{V}(x)=\left(1+x+x^{2}\right)\left(1+x^{3}\right)\left(1+x^{6}\right)
$$

Then there are nine possible transmit-receive array designs:

$$
\begin{aligned}
& P_{T}(x)=(1+x), \quad P_{R}(x)=\left(1+x^{2}\right)\left(1+x^{4}+x^{8}\right), \\
& P_{T}(x)=\left(1+x^{2}\right), \quad P_{R}(x)=(1+x)\left(1+x^{4}+x^{8}\right), \\
& P_{T}(x)=\left(1+x^{4}+x^{8}\right), \quad P_{R}(x)=\left(1+x^{2}\right)(1+x), \\
& P_{T}(x)=(1+x), \quad P_{R}(x)=\left(1+x^{2}+x^{4}\right)\left(1+x^{6}\right), \\
& P_{T}(x)=\left(1+x^{2}+x^{4}\right), \quad P_{R}(x)=(1+x)\left(1+x^{6}\right),
\end{aligned}
$$

$$
\begin{aligned}
& P_{T}(x)=\left(1+x^{6}\right), \quad P_{R}(x)=(1+x)\left(1+x^{2}+x^{4}\right), \\
& P_{T}(x)=\left(1+x+x^{2}\right), \quad P_{R}(x)=\left(1+x^{3}\right)\left(1+x^{6}\right),
\end{aligned}
$$

$$
\begin{array}{ll}
P_{T}(x)=\left(1+x^{3}\right), & P_{R}(x)=\left(1+x+x^{2}\right)\left(1+x^{6}\right), \\
P_{T}(x)=\left(1+x^{6}\right), & P_{R}(x)=\left(1+x+x^{2}\right)\left(1+x^{3}\right) .
\end{array}
$$

Suppose that the number of the training signals is $K=64$; Figure 6 shows the possible nine transmitter-receiver arrays. It can be noticed that 8 antennas are required for the transmitreceive pairs (a), (b), (d), (f), (h), and (i), and 7 antennas are required for the transmit-receive pairs (c), (e), and (g). All of the nine transmitter-receiver arrays have 10 degrees-offreedom. Figure 7 gives the statistical mean and STD of the 


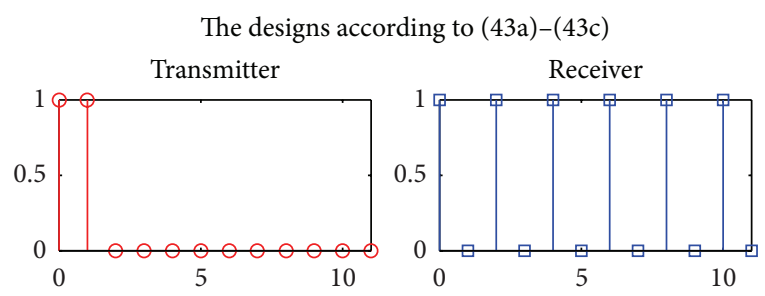

(a)
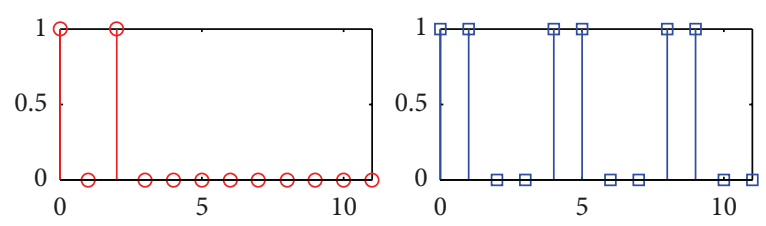

(b)

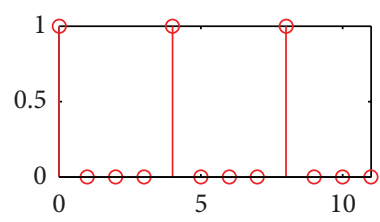

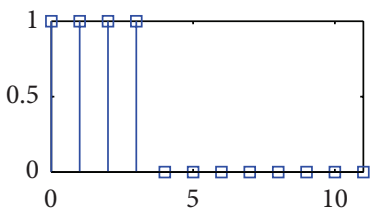

(c)

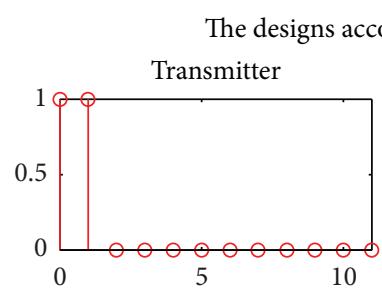

(d)
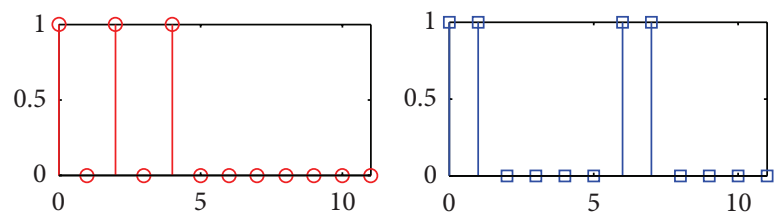

(e)
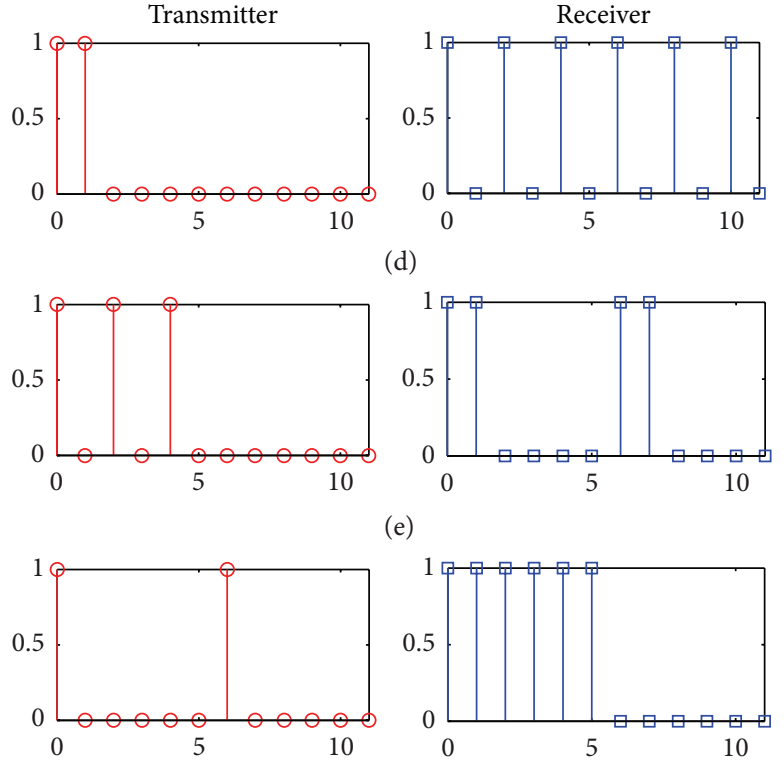

d)

(f)

The designs according to (43g)-(43i)
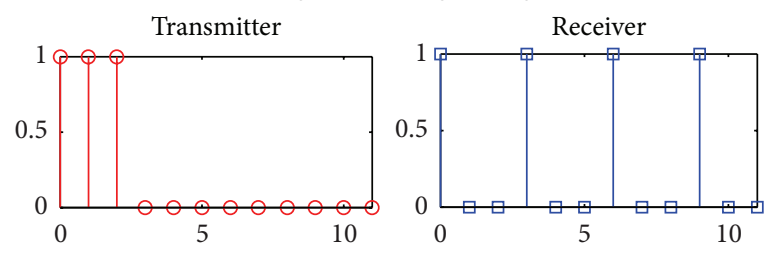

(g)
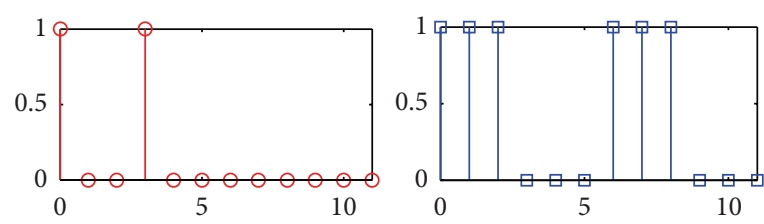

(h)
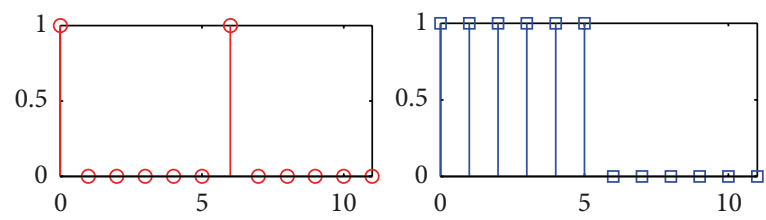

(i)

FIGURE 6: Illustration of the nine possible transmit-receive array positions for the MIMO antenna array. The $x$ axis stands for the MIMO antenna position. $y=1$ denotes an antenna and $y=0$ means no antenna.

output SINR performance. Since they have the same number of degrees-of-freedom, the nine transmit-receive arrays have the same statistical SINR performance.

\section{Conclusion}

In MIMO antenna array systems, the transmitter and receiver should be carefully designed to fully utilize the virtual MIMO antenna array. In this paper, we designed the MIMO antenna array with the polynomial factorization method and comparatively analyzed the designed array performance. For a desired virtual antenna array, the polynomial factorization method can optimally design the specified transmitter and receiver antenna arrays. Design examples are also provided, which are verified by simulation results. Note that two MIMO antenna arrays may have the same statistical SINR performance; they often should be seen as two different MIMO antenna arrays in the design because they have different transmit array gain and receive array gain. In this paper, uniform virtual MIMO antenna array elements are assumed. 


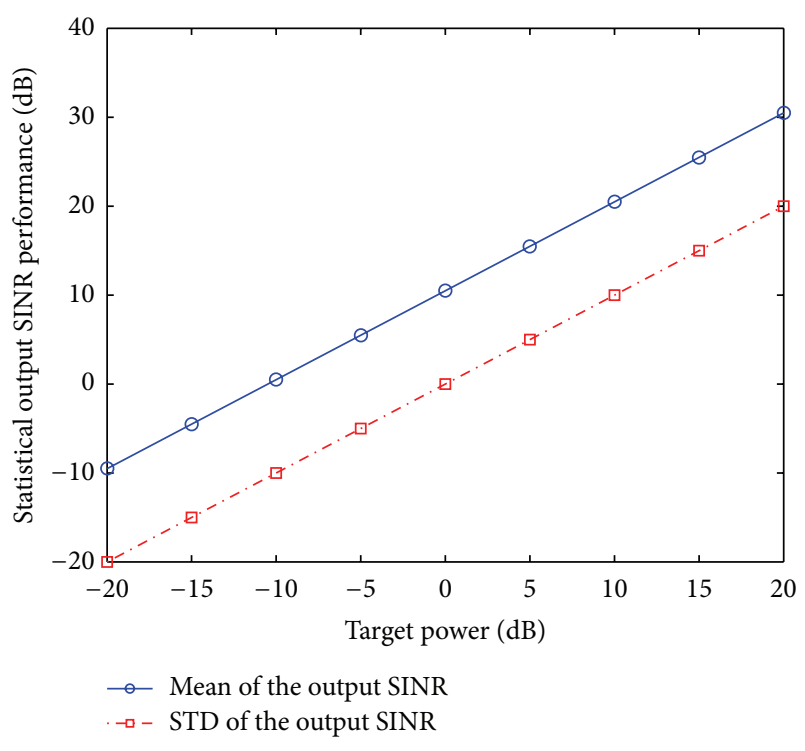

FIGURE 7: Statistical output SINR performance.

We are aware that nonuniform virtual MIMO antenna array elements may be desired in actual MIMO antenna arrays. This topic will be further investigated in our subsequent work.

\section{Acknowledgments}

This work was supported in part by the National Natural Science Foundation of China under Grant no. 41101317, the Fundamental Research Funds for the Central Universities under Grant no. ZYGX2010J001, and the Program for New Century Excellent Talents in University under Grant no. NCET-12-0095.

\section{References}

[1] W. Chen, X. Wang, and Z. Feng, "Design of compact dualpolarized antennas for MIMO handsets," International Journal of Antennas and Propagation, vol. 2012, Article ID 954742, 8 pages, 2012.

[2] J. Zheng, Y. Li, and Z. Feng, "Impact of mutual coupling and polarization of antennas on BER performances of spatial multiplexing MIMO systems," International Journal of Antennas and Propagation, vol. 2012, Article ID 795205, 12 pages, 2012.

[3] Q. Zeng, Y. Yao, S. Liu, J. Yu, P. Xie, and X. Chen, “Tetraband small-size printed strip MIMO antenna for mobile handset application," International Journal of Antennas and Propagation, vol. 2012, Article ID 320582, 8 pages, 2012.

[4] C. Yang, Y. Yao, J. Yu, and X. Chen, "Novel compact multiband MIMO antenna for mobile terminal," International Journal of Antennas and Propagation, vol. 2012, Article ID 691681, 9 pages, 2012.

[5] R. K. Mehra, "Optimal input signals for parameter estimation in dynamic systems-survey and new results," IEEE Transactions on Automatic Control, vol. 19, no. 6, pp. 753-768, 1974.

[6] J. Yang and S. Roy, "On joint transmitter and receiver optimization for multiple-input-multiple-output (MIMO) transmission systems," IEEE Transactions on Communications, vol. 42, no. 12, pp. 3221-3231, 1994.

[7] L. Vandendorpe and O. van de Wiel, "MIMO DFE equalization for multitone DS/SS systems over multipath channels," IEEE Journal on Selected Areas in Communications, vol. 14, no. 3, pp. 502-511, 1996.

[8] L. Vandendorpe, J. Louveaux, B. Maison, and A. Chevreuil, "About the asymptotic performance of MMSE MIMO DFE for filter-bank based multicarrier transmission," IEEE Transactions on Communications, vol. 47, no. 10, pp. 1472-1475, 1999.

[9] J. Li and P. Stoica, MIMO Radar Signal Processing, John Wiley \& Sons, New York, NY, USA, 2009.

[10] W. Q. Wang, "Space-time coding MIMO-OFDM SAR for high-resolution imaging," IEEE Transactions on Geoscience and Remote Sensing, vol. 49, no. 8, pp. 3094-3104, 2011.

[11] W. Q. Wang and J. Y. Cai, "MIMO SAR using chirp diverse waveform for wide-swath remote sensing," IEEE Transactions on Aerospace and Electronic Systems, vol. 48, no. 4, pp. 3171-3185, 2012.

[12] W. Q. Wang, Multi-Antenna Synthetic Aperture Radar, CRC Press, New York, NY, USA, 2013.

[13] M. Bachmann, M. Schwerdt, and B. Bräutigam, "Accurate antenna pattern modeling for phased array antennas in SAR applicationsłdemonstration on TerraSAR-X," International Journal of Antennas and Propagation, vol. 2009, Article ID 492505, 9 pages, 2009.

[14] W. Wang, "Virtual antenna array analysis for MIMO synthetic aperture radars," International Journal of Antennas and Propagation, vol. 2012, Article ID 587276, 10 pages, 2012.

[15] D. K. C. So and Y. Lan, "Virtual receive antenna for overloaded MIMO layered space-time system," IEEE Transactions on Communications, vol. 60, no. 6, pp. 1610-1620, 2012.

[16] B. Friedlander, "On the relationship between MIMO and SIMO radars," IEEE Transactions on Signal Processing, vol. 57, no. 1, pp. 394-398, 2009.

[17] D. Fuhrmann, J. P. Browning, and M. Rangaswamy, "Signaling strategies for the hybrid MIMO phased-array radar," IEEE Journal on Selected Topics in Signal Processing, vol. 4, no. 1, pp. 66-78, 2010.

[18] W. Q. Wang and H. Z. Shao, "A flexible phased-MIMO array antenna with transmit beamforming," International Journal of Antennas and Propagation, vol. 2012, Article ID 609598, 10 pages, 2012.

[19] D. R. Fuhrmann and G. San Antonio, "Transmit beamforming for MIMO radar systems using signal cross-correlation," IEEE Transactions on Aerospace and Electronic Systems, vol. 44, no. 1, pp. 171-186, 2008.

[20] S. Ahmed, J. S. Thompson, Y. R. Petillot, and B. Mulgrew, "Finite alphabet constant-envelope waveform design for MIMO radar," IEEE Transactions on Signal Processing, vol. 59, no. 11, pp. 53265337, 2011.

[21] C. Z. Ma, T. S. Yeo, C. S. Tan, Y. Qing, and T. Zhang, "Receiver design for MIMO radar range sidelobe suppression," IEEE Transactions on Signal Processing, vol. 58, no. 10, pp. 5469-5474, 2010.

[22] W. Q. Wang, "Large-area remote sensing in high-altitude highspeed platform using MIMO SAR," IEEE Journal of Selected Topics in Applied Earth Observation and Remote Sensing, vol. 6, no. 99, pp. 1-13, 2013.

[23] W. T. Li, Y. Q. Hei, J. Yang, and X. W. Shi, "Fractal-based thinned planar-array design utilizing iterative FFT technique," 
International Journal of Antennas and Propagation, vol. 2012, Article ID 329648, 7 pages, 2012.

[24] C. C. Chang, R. H. Lee, and T. Y. Shih, "Design of a beam switching/steering butler matrix for phased array system," IEEE Transactions on Antennas and Propagation, vol. 58, no. 2, pp. 367-374, 2010.

[25] H. T. Chou, K. L. Hung, and H. H. Chou, "Design of periodic antenna arrays with the excitation phases synthesized for optimum near-field patterns via steepest descent method," IEEE Transactions on Antennas and Propagation, vol. 59, no. 11, pp. 4342-4345, 2011.

[26] M. Elmer, B. D. Jeffs, K. F. Warnick, J. R. Fisher, and R. D. Norrod, "Beamformer design methods for radio astronomical phased array feeds," IEEE Transactions on Antennas and Propagation, vol. 60, no. 2, pp. 903-914, 2012.

[27] R. M. Leahy and B. D. Jeffs, "On the design of maximally sparse beamforming arrays," IEEE Transactions on Antennas and Propagation, vol. 39, no. 8, pp. 1178-1187, 1991.

[28] S. Holm, B. Elgetun, and G. Dahl, "Properties of the beampattern of weight- and layout-optimized sparse arrays," IEEE Transactions on Ultrasonics, Ferroelectrics, and Frequency Control, vol. 44, no. 5, pp. 983-991, 1997.

[29] R. L. Haupt, “Thinned arrays using genetic algorithms," IEEE Transactions on Antennas and Propagation, vol. 42, no. 7, pp. 993-999, 1994.

[30] A. Austeng and S. Holm, "Sparse 2-D arrays for 3-D phased array imaging-design methods," IEEE Transactions on Ultrasonics, Ferroelectrics, and Frequency Control, vol. 49, no. 8, pp. 10731086, 2002.

[31] M. Donelli, A. Martini, and A. Massa, "A hybrid approach based on PSO and hadamard difference sets for the synthesis of square thinned arrays," IEEE Transactions on Antennas and Propagation, vol. 57, no. 8, pp. 2491-2495, 2009.

[32] S. K. Mitra, K. Mondal, M. K. Tchobanou, and G. J. Dolecek, "General polynomial factorization-based design of sparse periodic linear arrays," IEEE Transactions on Ultrasonics, Ferroelectrics, and Frequency Control, vol. 57, no. 9, pp. 1952-1966, 2010.

[33] S. K. Mitra, M. K. Tchobanou, and M. I. Bryukhanov, "A general method for designing sparse antenna arrays," in Proceedings of the 17th European Conference Circuit Theory and Design, vol. 2, pp. 263-266, Cork, Ireland, September 2005.

[34] W. J. LeVeque, Fundamentals of Number Theory, AddisonWesley, Reading, Mass, USA, 1977.

[35] S. K. Mitra, M. K. Tchobanou, and G. Jovanovic-Dolecek, "A simple approach to the design of one-dimensional sparse arrays," in Proceedings of the IEEE International Symposium on Circuits \& Systems, vol. 3, pp. 541-544, Vancouver, Canada, May 2004.

[36] S. K. Mitra, G. J. Dolecek, and M. K. Tchobanou, "On the design of one-dimensional sparse arrays with apodized end elements," in Proceedings of the European Signal Processing Conference, pp. 2239-2241, Vienna, Austria, September 2004.

[37] H. L. Van Trees, Optimum Array Processing, Wiley, New York, 2002.

[38] I. S. Reed, J. D. Mallett, and L. E. Brennan, "Rapid convergence rate in adaptive arrays," IEEE Transactions on Aerospace and Electronic Systems, vol. 10, no. 6, pp. 853-863, 1974.

[39] J. Capon and N. R. Goodman, "Probability distributions for estimators of the frequency-wavenumber spectrum," Proceedings of the IEEE, vol. 58, no. 10, pp. 1785-1786, 1970. 

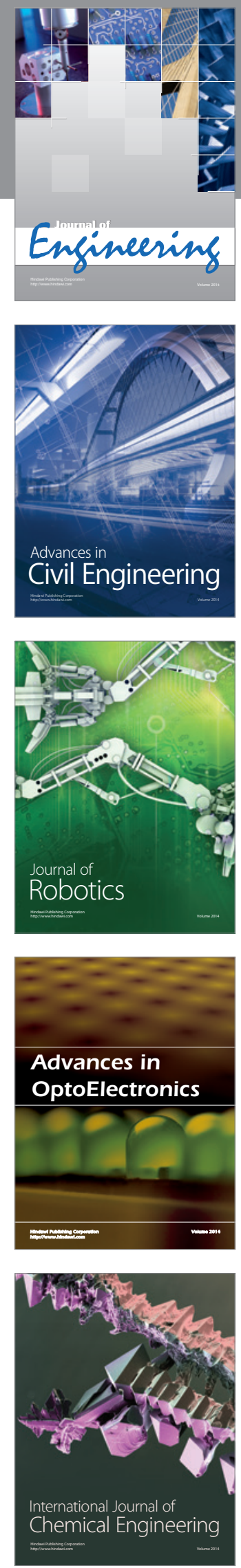

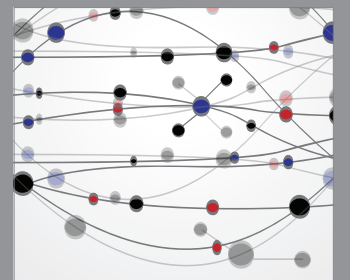

The Scientific World Journal
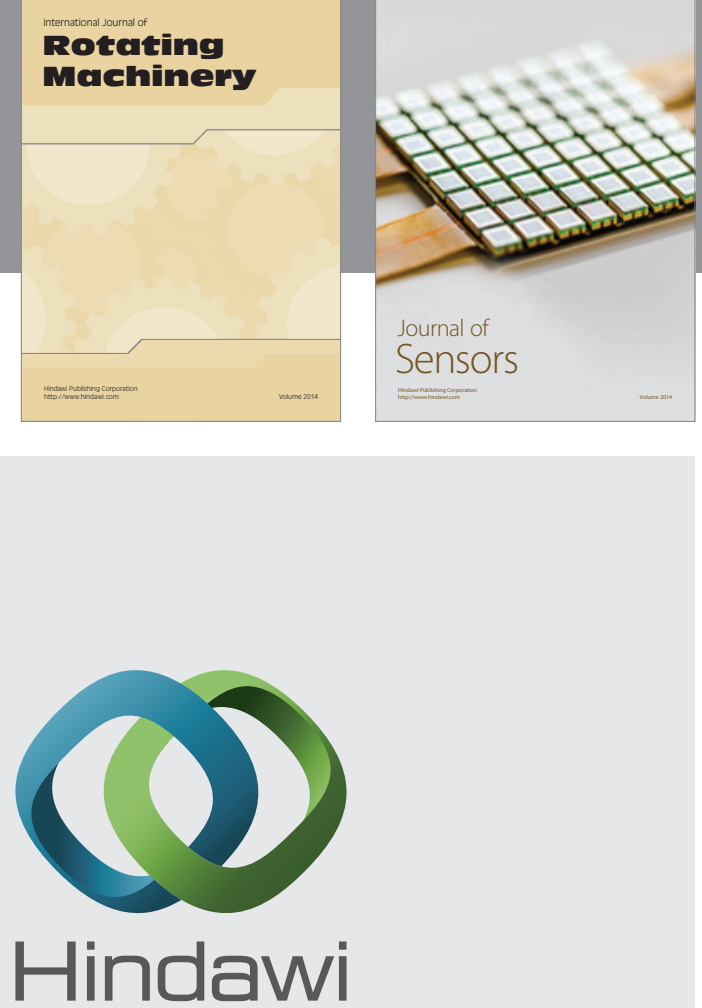

Submit your manuscripts at http://www.hindawi.com
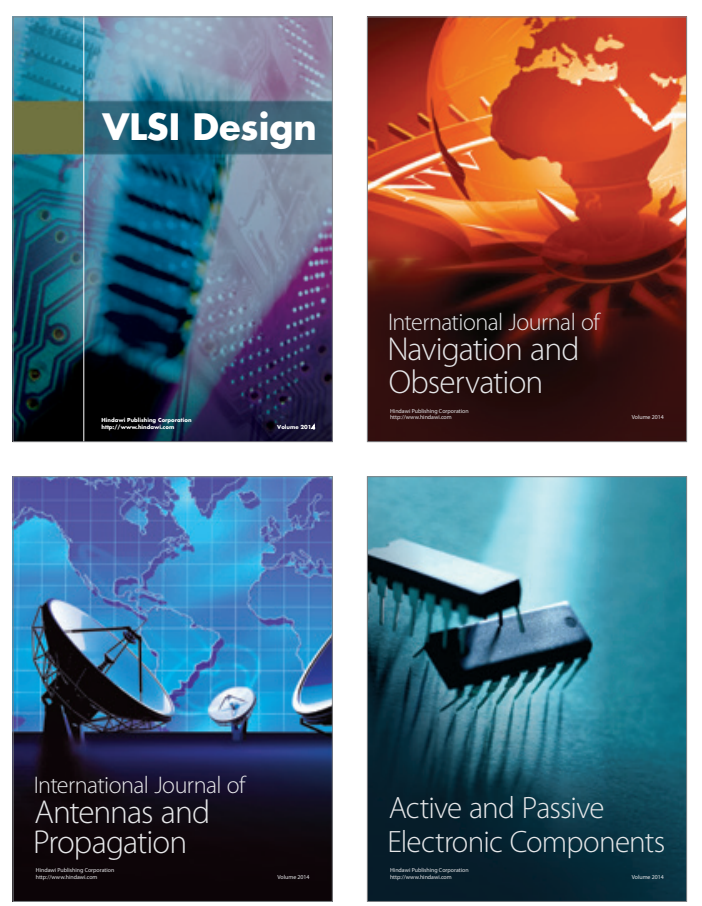
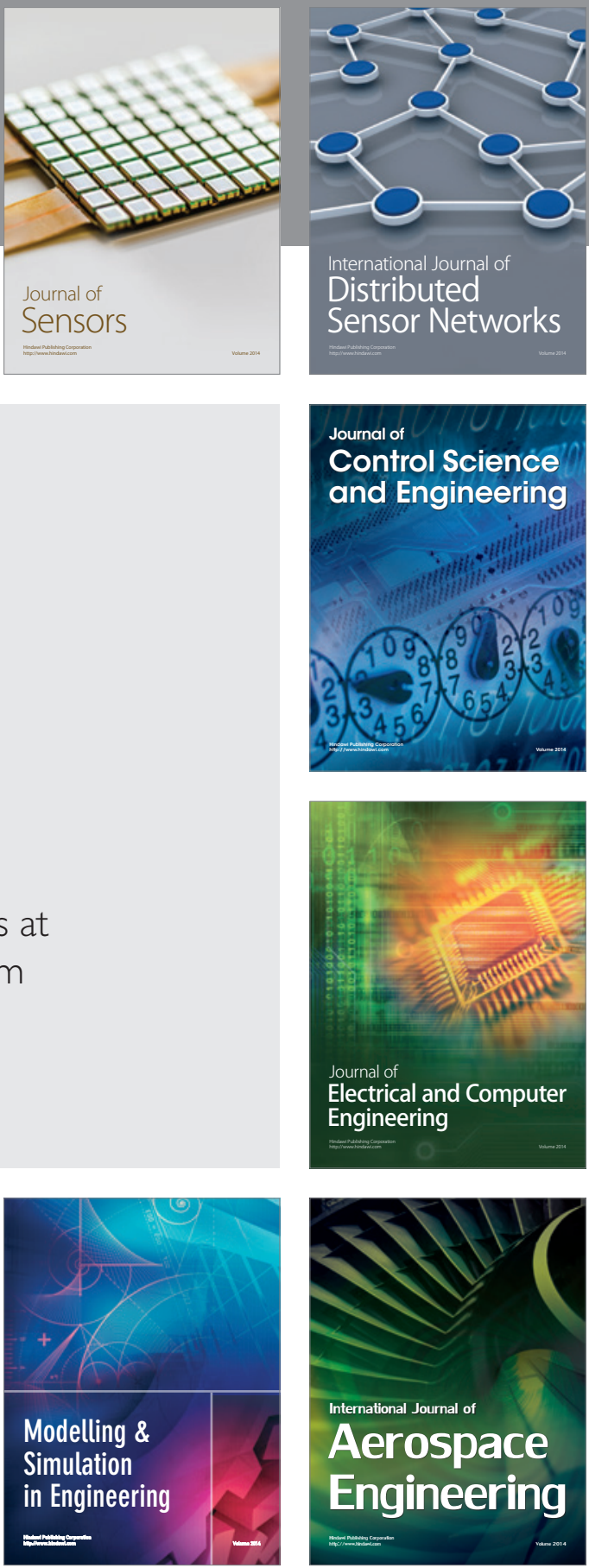

Journal of

Control Science

and Engineering
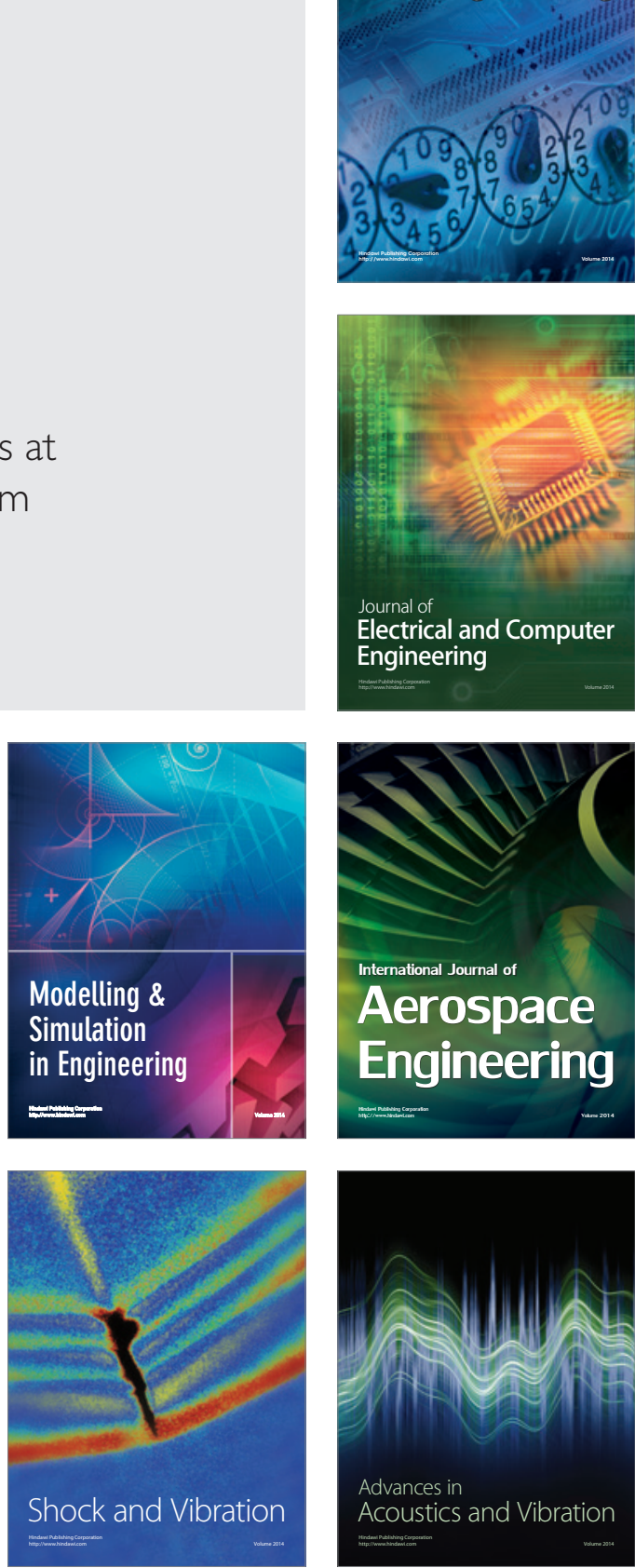